\title{
Management of Gonarthrosis with a Rotating Hinge Prosthesis: Minimum 10-Year Follow-up
}

\author{
Daniel Kendoff, MD, Carl Haasper, MD, Thorsten Gehrke, MD, \\ Wolfgang Klauser, MD, Nemandra Sandiford, FRCS (Tr/Orth)* \\ Orthopaedic Department, ENDO-Klinik, Hamburg, Germany, \\ *Joint Reconstruction Unit, Southland Hospital, Invercargill, New Zealand
}

\begin{abstract}
Background: The use of hinged designs is usually reserved for severe deformities or instability in contemporary total knee arthroplasty (TKA). Results have been mixed with some authors reporting relatively high incidences of complications. The aim of this study is to present the results of primary TKA performed with a hinged prosthesis with a minimum 10-year follow-up. We also examined the factors that influence survivorship of this prosthesis.

Methods: A total of 238 primary TKA procedures were performed using hinged prostheses. Indications included osteoarthritis, rheumatoid arthritis, posttraumatic deformity, and arthritis. Clinical outcomes were assessed using the Hospital for Special Surgery score. Radiologic assessment was performed at each follow-up. Survivorship was calculated based on the Kaplan-Meier method. All complications were documented.

Results: Mean follow-up was 13.5 years (standard deviation [SD], 3.4). Mean flexion at final review was $118^{\circ}\left(S D, 20^{\circ}\right)$. Fifty-four percent and $20 \%$ reported excellent and good functional scores, respectively. Survivorship was $94 \%$ at 13.5 years in patients over 60 years of age and $77 \%$ in patients less than 60 years of age. Survivorship in patients with preoperative varus deformity was $96 \%$ and that in valgus knees was $79 \%$.

Conclusions: The results of this study suggest that when rotating hinges are used for primary TKA, the best results are achieved in patients over 60 years old. The indications for this design in the setting of primary TKA include significant deformities, severe bone loss, and ligamentous laxity.
\end{abstract}

Keywords: Hinge, Knee replacement, Rotating hinge, Valgus, Deformity

The main indications for rotating hinge designs in the setting of primary total knee arthroplasty (TKA) are significant deformity or gross instability or both. ${ }^{1-4)}$ The use of unconstrained prostheses in these settings has been associated with an increased incidence of complications including poor soft-tissue balance and instability. ${ }^{5)}$ The Endo Model Rotating Hinge Design (Waldemar Link, Hamburg, Germany) has been used extensively in our surgical units

Received November 5, 2019; Accepted March 31, 2020

Correspondence to: Nemandra Sandiford, FRCS (Tr/Orth)

Joint Reconstruction Unit, Southland Hospital, 20 Holywood Lane, Gladstone, Invercargill 9810, New Zealand

Tel: +64-279306064

E-mail: nemsandiford@gmail.com in cases in which a hinged prosthesis was required for primary TKA. ${ }^{6-8)}$ Encouraging mid-term results have been reported with the use of this prosthesis from the designer institution ${ }^{8)}$ as well as other centres. ${ }^{9)}$ Some authors have reported worrying complication rates with survivorship of $88.7 \%$ at 1 year after surgery ${ }^{10)}$ with the use of the rotating hinge as a primary prothesis. Mortazavi et al. ${ }^{10)}$ recommended this design for revision procedures as opposed to primary TKA due to the high incidence of complications, which they encountered.

The aim of this study was to present the clinical and radiologic outcomes of the Endo Model rotating hinge prosthesis used for primary TKA with a minimum 10-year follow-up. We also examined anatomical and demographic factors that influence survival of this prosthesis. 


\section{METHODS}

A retrospective study was performed. A total of 238 primary TKAs were performed in 238 patients using the Endo Model Rotating Hinge Prosthesis (Waldemar Link) (Fig. 1) in 2008. One hundred and eighty-nine females and 49 males were included. The indications for the use of rotating hinged prostheses in primary TKA were advanced deformity (150 cases), significant bone loss (48 cases), and ligamentous laxity (40 cases). A total of 650 primary TKA procedures were performed in our unit that year. The mean age of patients was 67 years (range, 26-88 years). The prosthesis was inserted in 130 right and 108 left knees.

Indications for surgery included osteoarthritis (n $=208,88 \%)$, rheumatoid arthritis $(\mathrm{n}=12,5 \%)$, posttraumatic arthritis $(\mathrm{n}=12,5 \%)$, osteonecrosis $(\mathrm{n}=3,1 \%)$, and tumors $(\mathrm{n}=3,1 \%)$. The average American Society of Anesthesiologists (ASA) score was 3 (range, 2-4) (Table 1).

Sixty-two patients (26\%) died due to causes unrelated to their knee replacement procedures and 16 patients (7\%) were lost to follow-up. One hundred and sixty patients were available for review. Nineteen patients (12\%) had undergone revision surgery with prosthesis exchange at the time of final follow-up.

\section{Evaluation}

Survivorship was calculated using the Kaplan-Meier method. Patients who died or were lost to follow-up were censored.

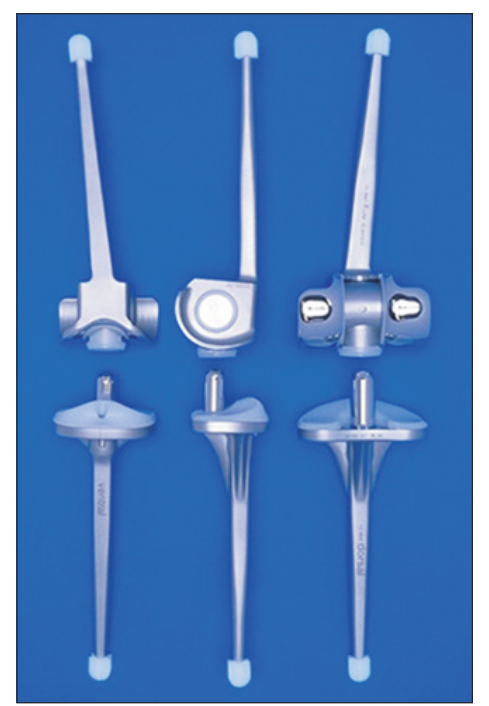

Fig. 1. First-generation Endo Modell Rotating Hinge prosthesis. The Monobloc prosthesis is made of a cobalt chromium molybdenum alloy. A trochlear flange was not included in the first-generation design, which was the subject of this paper.

\section{Surgical Technique}

All procedures were performed by 6 consultants and 3 residents. A tourniquet was used in all cases. It was only inflated during cementation. A subvastus approach was used in all cases. The patella was not resurfaced in any case. The femoral attachments of the collateral ligaments were released during the surgical exposure. This was performed in order to optimize surgical exposure while minimizing iatrogenic damage to the ligaments and surrounding bone. If necessary, the popliteus tendon was divided and the lateral head of the gastrocnemius divided in large fixed valgus deformities.

One dose of prophylactic antibiotics was administered and low-molecular-weight heparin was used for thromboprophylaxis. A suction drain was used for 48 hours after surgery in all cases. Patients were allowed to fully weight bear and mobilize on day 1 after surgery. Average length of stay was 14 days. The cemented longstemmed Endo Model Rotating Hinge prosthesis without an anterior femoral flange (Waldemar Link) (Fig. 1) was used in all cases. At this time this was the prosthesis of choice in the senior author's institution. Patients were assessed preoperatively as well as 2 weeks, 6 weeks, and annually thereafter. Clinical outcome was assessed using the Hospital for Special Surgery (HSS) score. This score is domain-specific and examines range of motion and level of pain experienced by the patient as well as functional level (ability to perform activities of daily living. The maximum

\section{Table 1. Patient Demographics}

\begin{tabular}{lcc}
\multicolumn{1}{c}{ Variable } & Baseline & Final follow-up \\
\hline Number & $280^{*}$ & $160^{*}$ \\
\hline Male : female & $49: 189$ & $125: 35$ \\
\hline Mean age (range, yr) & & $67(26-86)$ \\
\hline Average ASA score (range) & & $3(2-4)$ \\
\hline Number of patients revised & & 19 \\
\hline Procedure other than revision & & 9 \\
\hline Diagnoses & 208 & 136 \\
\hline OA & 12 & 7 \\
\hline Rheumatoid arthritis & 12 & 12 \\
\hline Post traumatic OA & 3 & 3 \\
\hline Osteonecrosis & 3 & 2 \\
\hline Tumor & & \\
\hline ASA:Amercan Socisty & & \\
\hline
\end{tabular}

ASA: American Society of Anesthesiologists, 0A: osteoarthritis. *Deceased, 62; Lost to follow-up, 16. 
Kendoff et al. Rotating Hinge in Primary Total Knee Arthroplasty

Clinics in Orthopedic Surgery • Vol. 12, No. 4, $2020 \bullet$ www.ecios.org

score is 100. A score of $<50$ is poor, 50-69 fair, 70-89 fair, and 90-100 excellent. Patient satisfaction was assessed using a visual analog scale with a rating system of poor, fair, good, and excellent.

Radiographic assessment was performed preoperatively and at each follow-up visit. Each radiographic image was reviewed by 2 surgeons (DK and TG) looking for signs of loosening at the bone prosthesis and cement prosthesis interface as well as alignment of the prosthesis. Signs of loosening were considered to be present if there was a radiolucent line at the cement prosthesis interface or the cement bone interface of the femoral or tibial components, which was greater than $2 \mathrm{~mm}$, or if there were progressive radiolucent lines on serial radiographic examinations. ${ }^{11)}$

\section{RESULTS}

Mean follow up was 13.5 years (range, 13-14.5 years). Sixty-two patients died due to causes unrelated to their knee operations. None of these patients had complaints relating to their replaced knee. Sixteen patients (9\%) were lost to follow-up as they had emigrated prior to their 10-year follow-up. Up to the point at which they emigrated, they had no complaints regarding their operated knees. One hundred and sixty patients were thus available for clinical follow-up. Nineteen patients (12\%) underwent a revision procedure.

Eighty-six patients (54\%) reported excellent results and $32(20 \%)$ reported good results. Fair and poor results were reported by $19(12 \%)$ and $22(14 \%)$ patients, respectively. Review of the results based on the visual analog scale for patient satisfaction revealed that $125(78 \%)$ of patients reported excellent results, $25(16 \%)$ reported good results, $5(3 \%)$ reported fair and $5(3 \%)$ reported poor results. There was no evidence of progressive radiolucent lines around any component, radiographic signs of loosening, or impending revisions at the time of final followup. Mean arc of flexion at final review was $118^{\circ}$ (standard deviation, $20^{\circ}$ ).

\section{Revisions}

Nineteen patients underwent revision procedures. Of these, $6(32 \%)$ were for patella wear requiring patella resurfacing and $5(26 \%)$ required revision due to deep infection. Three (16\%) required revision for mechanical failure of the rotating hinge mechanism, 2 (11\%) for dissociation of the hinge mechanism, 1 (5\%) for aseptic loosening, 1 (5\%) for extensor mechanism disruption, and 1 (5\%) for a traumatic femoral fracture. All patients in this group required either partial or complete exchange of their pros- theses or addition of a patellar component.

Nine patients underwent further surgical procedures to the knee but did not have revision or exchange of TKA components. Three underwent patella debridement and denervation. Two underwent lateral release and medial plication for patella dislocation, 2 underwent excision of the inferior pole of the patella due to inferior pole fractures, one for open arthrolysis secondary to stiffness, and one for fixation of a distal femoral fracture.

The overall reoperation rate at the time of final follow-up including these 9 cases was $17.5 \%$. The incidence of revision of the tibial or femoral component of the hinge, which was inserted at the primary procedure, was $7 \%$.

\section{Implant Survivorship}

The overall survivorship at a mean of 13.5 years of followup was $88 \%$ with failure defined as a revision of the prosthesis for any reason (Fig. 2). When we analyzed revision rates based on the age of our population, we found that survivorship for those over 60 years of age was $94 \%$ at 13.5 years of follow-up. Survivorship over the same time period was $77 \%$ in those under 60 years (Fig. 3). We also examined survivorship in relation to preoperative limb alignment. Patients with a preoperative varus alignment had survivorship of $96 \%$ at 13.5 years compared to $79 \%$ at the same duration of follow-up in those with a preoperative valgus deformity (Fig. 4).

Component survivorship in patients who were under 60 years of age and had a preoperative valgus deformity was $64 \%$ at 13.5 years. There was no difference in survivorship based on sex, previous surgery to the re-

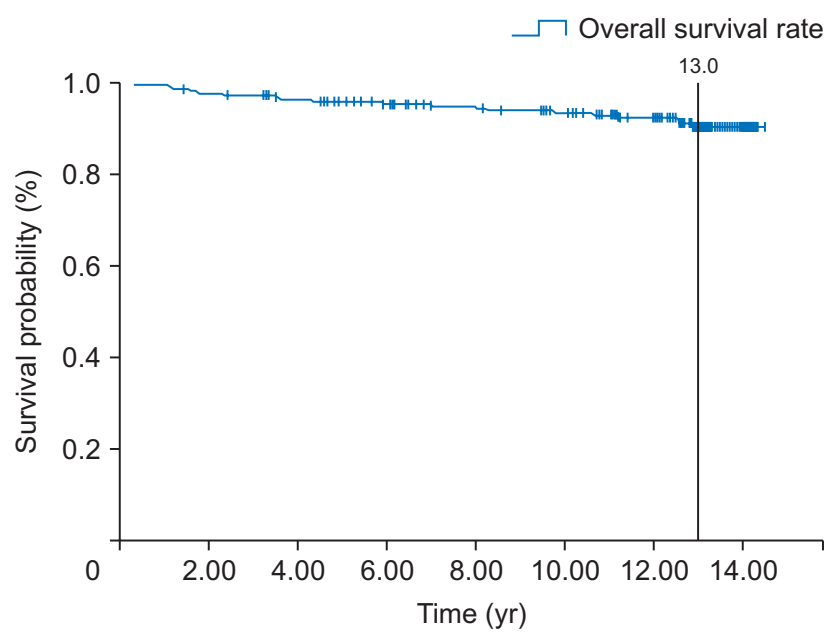

Fig. 2. Kaplan-Meier graph illustrating overall survivorship for the entire cohort. Patients who died or were lost to follow-up were censored. Survivorship at 13.5 year is $88 \%$. 
Kendoff et al. Rotating Hinge in Primary Total Knee Arthroplasty

Clinics in Orthopedic Surgery • Vol. 12, No. 4, $2020 \bullet$ www.ecios.org

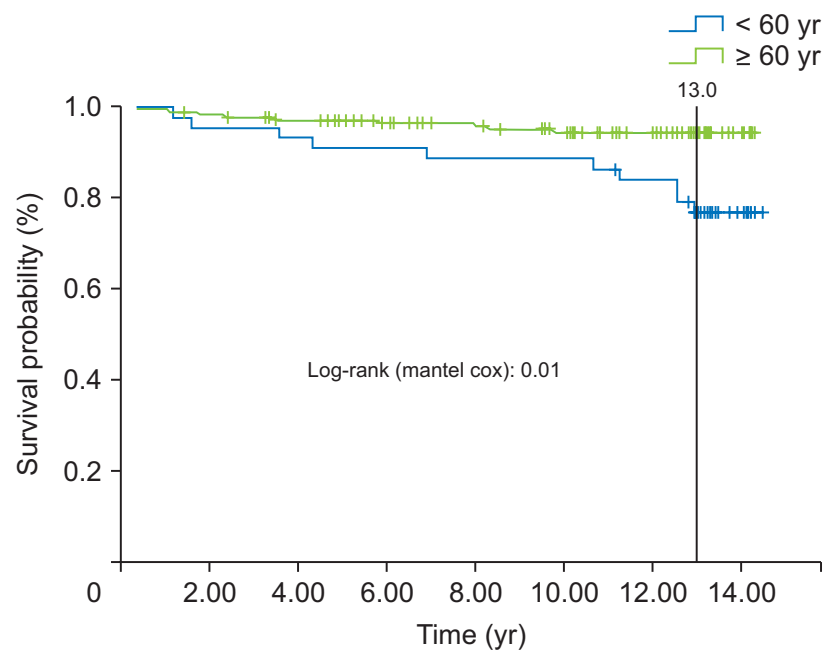

Fig. 3. Kaplan-Meier graph illustrating survivorship of patients based on age $(<60$ and $\geq 60 \mathrm{yr})$.

placed knee (arthroscopies or menisectomies), or the primary pathology (osteoarthritis, posttraumatic arthritis, or inflammatory arthritis).

\section{DISCUSSION}

The most important findings of this study are that when rotating hinges were used for primary TKA, the best results were achieved in patients over 60 years old with preoperative varus deformities. The main indications for the use of rotating hinged prostheses in primary TKA include advanced deformity, which is often associated with bone loss and ligamentous laxity. This design has been used extensively in the first author's institution. ${ }^{7,8)}$

We were able to demonstrate in this series our 13year results of the use of the rotating hinge prosthesis for primary TKA. The results of this study suggest that good or excellent clinical and radiographic results can be achieved in the majority of patients with the use of a rotating hinge prosthesis in primary TKA. This is reflected by an overall survival rate of $88 \%$ in our patient cohort. Most interestingly, the survival rate was found to be superior in patients with preexisting varus deformities in combination with a related age above 60 years at the time of surgery. This cohort revealed a survival rate of above $95 \%$ after 13 years.

In contrast to these findings, the combination of a valgus deformity with a relative young age of implantation ( $<60$ years) did show a survival rate of only $64 \%$ after the same time period. It is possible that in this younger, more active population, the forces acting on the hinge mecha-

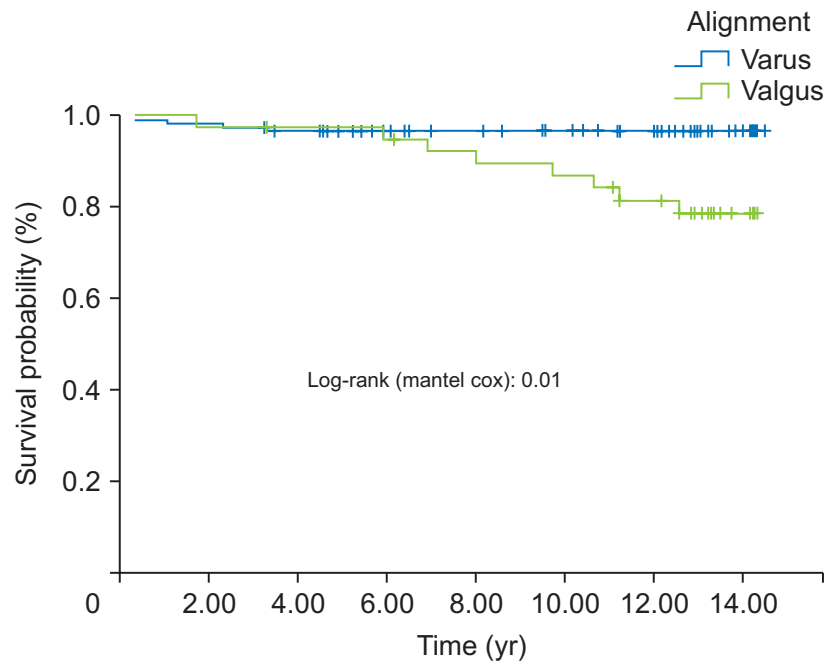

Fig. 4. Kaplan-Meier graph illustrating survivorship of patients based on preoperative knee alignment (varus and valgus).

nism and also the shear forces at the bone cement and prosthesis cement interfaces are higher leading to higher failure rates, but a clear reason has not be elucidated. Although our algorithm utilizing a rotating hinge implant in primary indications is rare in our current practice, we strictly avoid using a rotational hinge implant in younger patients with a severe valgus deformity and combined ligamentous instability. Based on these findings, we recommend the use of a fixed hinge implant in these patient groups in contrast to the literature. ${ }^{12)}$

The overall complication rate leading to any surgical revision was $11.5 \%$ after 13 years. This included an observed complication rate of $2 \%$ due to a deep infection and $3.5 \%$ due to secondary patellofemoral arthritis. This identified relevant patellofemoral limitation of the earlier implant design was based on the missing anterior femoral flange for an optimized patellar tracking. The design was subsequently changed to include an anterior flange for articulation with the patella. The current implant design has included this flange for the past 10 years. Over this time, we have observed that there has been a significant decrease in the incidence of symptomatic patellofemoral arthritis and need for secondary patella resurfacing.

The overall deep infection rate of $2 \%$ is consistent with most previous studies and with studies with comparable follow-up periods evaluating condylar unconstrained implants after 13 years. Based on the larger quantity of cement and intramedullary implant fixation, one might expect a higher infection rate, compared to "conventional" condylar type TKA implants. This, however, was not observed in our patient population and has been confirmed 
Kendoff et al. Rotating Hinge in Primary Total Knee Arthroplasty

Clinics in Orthopedic Surgery • Vol. 12, No. 4, $2020 \bullet$ www.ecios.org

by other authors and institutions. A study by Guenoun et al. ${ }^{13)}$ from 2009 revealed an unacceptably high complication rate of $>30 \%$ with the use of the rotating hinge prosthesis in primary indications and $>24 \%$ in revision cases. In addition, reported infection rates in these groups were significant with $11 \%$ in primary indications and $9 \%$ in revisions, respectively. Interestingly, the overall rate of aseptic loosening was found to be acceptable with $3.5 \%$ after 3 years of follow-up. The reasons for these findings are not clear. Such a reported high general complication rate needs to be monitored closely. It is possible that this is not related to a specific implant design. ${ }^{14-16)}$ Wear and osteolysis as in traditional TKA after 13 years were not found in our cohort.

Evaluating the clinical outcomes measures of our patients, most interestingly, we found inconsistency between the measured results of the HSS score and the patients' self-assessment. ${ }^{17)}$ While the HSS scores revealed over 20\% of patients had either fair or poor results, the patients' selfassessment revealed only 3\% had fair and 3\% had poor results with $>90 \%$ having excellent and good results. Our study has some limitations. This was a retrospective evaluation with no available comparative patient or implant group $^{18)}$ and $7 \%$ of all initial patients were lost to follow-up for various reasons. The results of this study suggest that good clinical and radiological results can be obtained with the use of rotating hinge prostheses in patients over 60 years of age and those with preoperative varus deformities. Survivorship is also encouraging in this group with the use of rotating hinge prostheses.

\section{CONFLICT OF INTEREST}

No potential conflict of interest relevant to this article was reported.

\section{REFERENCES}

1. Nelson CL, Gioe TJ, Cheng EY, Thompson RC Jr. Implant selection in revision total knee arthroplasty. J Bone Joint Surg Am. 2003;85 Suppl 1:S43-51.

2. Kurtz SM, Lau E, Ong K, Zhao K, Kelly M, Bozic KJ. Future young patient demand for primary and revision joint replacement: national projections from 2010 to 2030. Clin Orthop Relat Res. 2009;467(10):2606-12.

3. Kurtz SM, Ong KL, Lau E, Bozic KJ, Berry D, Parvizi J. Prosthetic joint infection risk after TKA in the Medicare population. Clin Orthop Relat Res. 2010;468(1):52-6.

4. Kowalczewski J, Marczak D, Synder M, Sibinski M. Primary rotating-hinge total knee arthroplasty: good outcomes at mid-term follow-up. J Arthroplasty. 2014;29(6):1202-6.

5. Zmistowski B, Restrepo C, Kahl LK, Parvizi J, Sharkey PF. Incidence and reasons for nonrevision reoperation after total knee arthroplasty. Clin Orthop Relat Res. 2011;469(1):13845 .

6. Lakstein D, Zarrabian M, Kosashvili Y, Safir O, Gross AE, Backstein D. Revision total knee arthroplasty for component malrotation is highly beneficial: a case control study. J Arthroplasty. 2010;25(7):1047-52.

7. Engelbrecht E, Nieder E, Strickle E, Keller A. Intracondylar knee joint prosthesis with rotation capacity: Endo model. Chirurg. 1981;52(6):368-75.

8. Nieder E. Sled prosthesis, rotating knee and hinge prosthesis: St. Georg model and ENDO-model: differential therapy in primary knee joint arthroplasty. Orthopade. 1991;20(3):170-80.

9. Petrou G, Petrou H, Tilkeridis C, et al. Medium-term results with a primary cemented rotating-hinge total knee replacement: a 7- to 15-year follow-up. J Bone Joint Surg Br. 2004;86(6):813-7.

10. Mortazavi SM, Schwartzenberger J, Austin MS, Purtill JJ, Parvizi J. Revision total knee arthroplasty infection: incidence and predictors. Clin Orthop Relat Res. 2010;468(8):2052-9.

11. Ewald FC. The Knee Society total knee arthroplasty roentgenographic evaluation and scoring system. Clin Orthop Relat Res. 1989;(248):9-12.

12. McAuley JP, Engh GA. Constraint in total knee arthroplasty: when and what? J Arthroplasty. 2003;18(3 Suppl 1):51-4.

13. Guenoun B, Latargez L, Freslon M, Defossez G, Salas N, Gayet LE. Complications following rotating hinge EndoModell (Link) knee arthroplasty. Orthop Traumatol Surg Res. 2009;95(7):529-36.

14. Barrack RL. Evolution of the rotating hinge for complex total knee arthroplasty. Clin Orthop Relat Res. 2001;(392):2929.

15. Singh JA, O'Byrne MM, Harmsen WS, Lewallen DG. Predictors of moderate-severe functional limitation 2 and 5 years after revision total knee arthroplasty. J Arthroplasty. 2010;25(7):1091-5.e1-4. 
Kendoff et al. Rotating Hinge in Primary Total Knee Arthroplasty

Clinics in Orthopedic Surgery • Vol. 12, No. 4, $2020 \bullet$ www.ecios.org

16. Ong KL, Lau E, Suggs J, Kurtz SM, Manley MT. Risk of subsequent revision after primary and revision total joint arthroplasty. Clin Orthop Relat Res. 2010;468(11):3070-6.

17. Bremner-Smith AT, Ewings P, Weale AE. Knee scores in a 'normal' elderly population. Knee. 2004;11(4):279-82.

18. Westrich GH, Mollano AV, Sculco TP, Buly RL, Laskin RS, Windsor R. Rotating hinge total knee arthroplasty in severly affected knees. Clin Orthop Relat Res. 2000;(379):195-208. 